\title{
Ageing Effects of Na-Alginate/PEO Spinning Solution on Electrospinnability and Morphology of Nanofibres
}

\author{
Mohammad Forhad Hossain, PhD \\ Mustafijur Rahman, MSc \\ Md. Golam Nur, BSc
}

Department of Wet Process Engineering, Bangladesh University of Textiles.

doi: 10.19044/esj.2017.v13n9p56 ～URL:http://dx.doi.org/10.19044/esj.2017.v13n9p56

\begin{abstract}
Poly(ethylene oxide) (PEO) loaded alginate nano-fibres were produced by electrospinning of the $4 \%(\mathrm{w} / \mathrm{w})$ of 70:30 Na-alginate/PEO spinning solution. In this research, the effects of solution viscosity and storage time of the spinning solution on fibre diameter and morphology were studied. Results show that the viscosity of the spinning solution was 2284 $\mathrm{cP}$ at the age of day 1 , which was dropped slowly and reached at $2184 \mathrm{cP}$ until day 5 . However, the viscosity sharply decreased after 5 days of storing and the mean diameter of the fibres remain similar until 20 days though the uniformity of the fibre diameters decreased significantly. At day 40, the viscosity of the spinning solution dropped $48 \mathrm{cP}$ which yields beads-onstring fibres. The solutions of 5 days and 10 days old produced smooth and uniform nanofibres having mean diameter of $133 \mathrm{~nm}$ and $132 \mathrm{~nm}$, respectively. They also produced stable electrospinning fibre jet compared to other solutions. Overall, the results revel that the electrospinnability of $\mathrm{Na}-$ alginate/PEO spinning solution remain suitable until 10 days of storing time.
\end{abstract}

Keywords: Electrospinning, spinnability, viscosity, nanofibres, fibre's diameter, morphology

\section{Introduction}

Electrospinning is the most useful and effective technique to make nano-fibres with controlled morphology from polymer solution. The electrospinning process is first introduced in the early 1930s, which is continuously investigated to date. This process is capable of producing ultrafine nano-scaled fibers by electrically charging a suspended droplet of polymer melt or solution (Reneker and Chun 1996). Due to action of a strong electrostatic force a fine fibre jet ejects from a capillary and travels through the air, and finally deposited on an electrically grounded collector (Son et al. 
2004). Electrospun nanofibres from biopolymers are used in biomedical applications such as tissue engineering, drug delivery and wound dressing, etc. However, the properties of polymer solution such as concentration, viscosity and solvent type greatly affect the spinnability and fibre morphology. The changes in the fibre diameter and morphology are investigated in respect of age and viscosity of the solution.

Alginate, a naturally derived biomaterial, produced from alginic acid which is extracted from seaweed. Structurally, the alginic acids are linier unbranched polymer similar to cellulose. This is a copolymer of $\alpha$-Lguluronic (G) acid and $\beta$-D-mannuronic (M) acid. Similar to other polysaccharide polymers sodium alginate solution is not suitable for electrospinning. This is because Na-alginate is a polyelectrolyte polymer which contains polyanions like chitosan ( $\mathrm{Li}$ and Hsieh 2006). The polyanions of Na-alginate molecule repulse each other and hinder molecular chain entanglement. Moreover, Na-alginate especially with high guluronic acid (G) content, which may turn into a gel easily, becomes too viscous for electrospinning at slightly higher concentration. These problems can be overcome by adding a suitable copolymer and a small amount of surfactant to the Na-alginate solution. Mincheva et al. (2005) found that the hydrogen bonds between the polyelectrolytes and non-ionic water-soluble copolymer poly(ethylene oxide) (PEO) favour electrospinning. Addition of a small amount of surfactant along with PEO reduces the surface tension and viscosity to improve the electro-spinnability of the spinning solution which allows increasing the alginate content in the solution (Saquing et al. 2013;Bhattarai et al. 2006). As a result, the spinnability and fibre formation of alginate/PEO would increase further, even at higher alginate content in the solution.

Previous studies (Saquing et al. 2013;Kong et al. 2009) have shown that a total polymer concentration of $4 \mathrm{wt} \%$ up to 70:30 Na-alginate/PEO yielded smooth nano-fibres. Hence, a 70:30 Na-alginate/PEO of $4 \mathrm{wt} \%$ solution is used for electrospinning experiments. Numerous studies (Baumgarten 1971;Demir et al. 2002;Deitzel et al. 2001;Jarusuwannapoom et al. 2005;Fong et al. 1999;Bhattarai et al. 2006) have investigated the effects of solution properties on fibre diameter and morphology. So far, very limited studies (Cho et al. 2012;Poursorkhabi et al. 2015) were carried out to investigate the effect of storage time (ageing) of spinning solution on fibre diameter and morphology. This study focuses the effects of ageing of spinning solution of Na-alginate/PEO on viscosity, fibre diameter and morphology. 


\section{Materials and methods \\ Materials}

Na-alginate powder is provided by ConvaTec UK, PEO powder $\left(M_{w}=900,000\right)$ and Triton X100 are purchased from Sigma-Aldrich, UK. The syringe $(10 \mathrm{ml})$ and needle $(18 \mathrm{G})$ are purchased from BD Plastipak, UK. All these polymer and reagents were used without further modification or purification.

\section{Preparation of Na-alginate/PEO spinning solution}

Predetermined amount of sodium alginate and PEO powder were dissolved in distilled water in a glass bottle. A small amount $(0.5 \%)$ of Triton X100 surfactant was added to increase the homogeneity of the solution. Then the sealed bottle is transferred to a magnetic stirrer and allowed gentle stirring at room temperature for 24 hours to obtain a homogeneous spinning solution. The prepared $4 \mathrm{wt} \%$ of 70:30 Na-alginate/PEO solution is then stored for 1 day in a dark place to remove the residual bubbles before spinning. The solution is stored in that dark place at room temperature for up to 40 days and electrospinning experiments were carried out according to a predetermined schedule.

\section{Methods}

\section{Electrospinning of Na-alginate/PEO}

A horizontal electrospinning device was used to make $\mathrm{Na}$ alginate/PEO nano-fibres. A syringe, loaded with spinning solution was set in the pump. The process parameters were adjusted to $16 \mathrm{~cm}$ working distance, $0.4 \mathrm{ml} / \mathrm{h}$ feed rate, and $10.5 \mathrm{kV}$ applied voltage to obtain uniform fibres. The electrospun fibres were collected on an aluminium foil. After allowing $1 \mathrm{~h}$ of electrospinning, the foil with deposited fibres was collected for drying 24 hours at room temperature to remove the residual solvents. The spinning solution was stored in a dark place for 40 days and electrospun fibres were prepared at day-1, day-5, day-10, day-20 and day-40. All of the electrospinning experiments were carried out at room temperature.

\section{Measurement of solution viscosity}

Properties of the polymer solution such as concentration and viscosity greatly affect the electrospinnability and fibre morphology. The solution with lower viscosity is not suitable for producing continuous fibres because of insufficient amount of materials in the solution. However, the viscosity of solution can be deteriorated due to prolonged storage. Sodium alginate and PEO both are biodegradable polymer and degrade during storage. Due to storing the spinning solution of Na-alginate/PEO might be degraded. This may change the viscosity of the solution, which ultimately 
affects the electrospinning process and morphology of the fibres. Thus, the age of the spinning solution is also an important factor for successful electrospinning of Na-alginate/PEO. The viscosity of the spinning solution is measured by Brookfield Viscometer (spindle size: SC4-34; spindle rpm: 20; model: LVDV-III+; manufacturer: BROOKFIELD Engineering Laboratories Inc., USA) at room temperature. The solution was stored in a dark place and measurements were taken at day- $1,-5,-10,-20$ and -40 at $25^{\circ} \mathrm{C}$.

\section{Characterization of electro-spun fibres}

The morphology and structure of the electrospunnano-fibres were observed by field emission gun scanning electron microscope (SEM) (PHILIPS XL30 FEG-SEM). The electrospun fibres mesh on aluminium foil were cut about $0.5 \mathrm{~cm} \times 0.5 \mathrm{~cm}$ size and adhered on a specimen stub by carbon tape specified for SEM purpose. Then the samples were coated with carbon using gatan Precision Etching Coating System (model: 682). The images were taken at 1000X, 2000X, 10,000X and 20,000X magnifications. The SEM operating parameters were set $6 \mathrm{kV}$ accelerating voltage and spot size 3 . The fibres diameters were manually measured by using the ImageJ software (ImageJ 2004) from 30 randomly selected fibres from the 10,000X and 20,000X magnification images at 3 different places. The ImageJ line drawing feature reports the line length in pixels. Pixels are converted to standard units of length measurements using the SEM image scale bar.

Fourier Transform Infrared Spectroscopy (FTIR) (model: NICOLET5700 FT-IR; manufacturer: Thermo Electron Corporation) was used to identify the chemical structure and molecular change occurred in the fibres. A typical FTIR records the frequencies on basis of absorption of infrared light related to respective chemical bonds. The infrared spectra of absorption mode of the samples were obtained from FTIR spectrometer which was connected with a PC. The scanning was carried out at room temperature within $4000 \mathrm{~cm}^{-1}$ to $400 \mathrm{~cm}^{-1}$ spectral range with 32 scans and a resolution of $4 \mathrm{~cm}^{-1}$. The scan was repeated and spectra were taken in three different places of each sample. Characterizing assessment was done on the nanofibres prepared using the solutions of age day-1, $-5,-10,-20$, and -40 .

\section{Results and discussion}

\section{Effect of ageing of solution on viscosity}

Viscosity of the spinning solution is one of the influential parameters which affects the structure and morphology of the fibres and the stability of electrospinning process (Tarun and Gobi 2012). Lower viscosities impede polymer chain entanglement and reduce viscoelastic force. As a result, the electrified solution jet cannot counter-balance the higher Coulombic stretching force during electrospinning, which results in fibre breaks, beads 
and instability of the process (Zhang and Hsieh 2008). However, too high viscosity can lead to difficulty in the initiation of fibre jet. The viscosity of polymeric solution could be decreased because of degradation of the constituent polymer during long time storage. Thus, the storing time of the spinning solution is also a considerable factor affecting the electrospun nanofibres. Figure 1 shows of the spinning solution at different ages and how the viscosity of a Na-alginate/PEO solution declines over the period of storage time. The highest viscosity was recorded $2284 \mathrm{cP}$ of the solution at the age of 1 day. Then the viscosities of the solution were decreased and recorded 2184, 1579, 202 and $48 \mathrm{cP}$ at the age of 5 days, 10 days, 20 days and 40 days, respectively. The viscosity of the Na-alginate/PEO solution decreased slowly until 5 days, and dropped sharply after that. This indicates the solution Na-alginate/PEO is unstable over the time of long storing and degraded eventually.

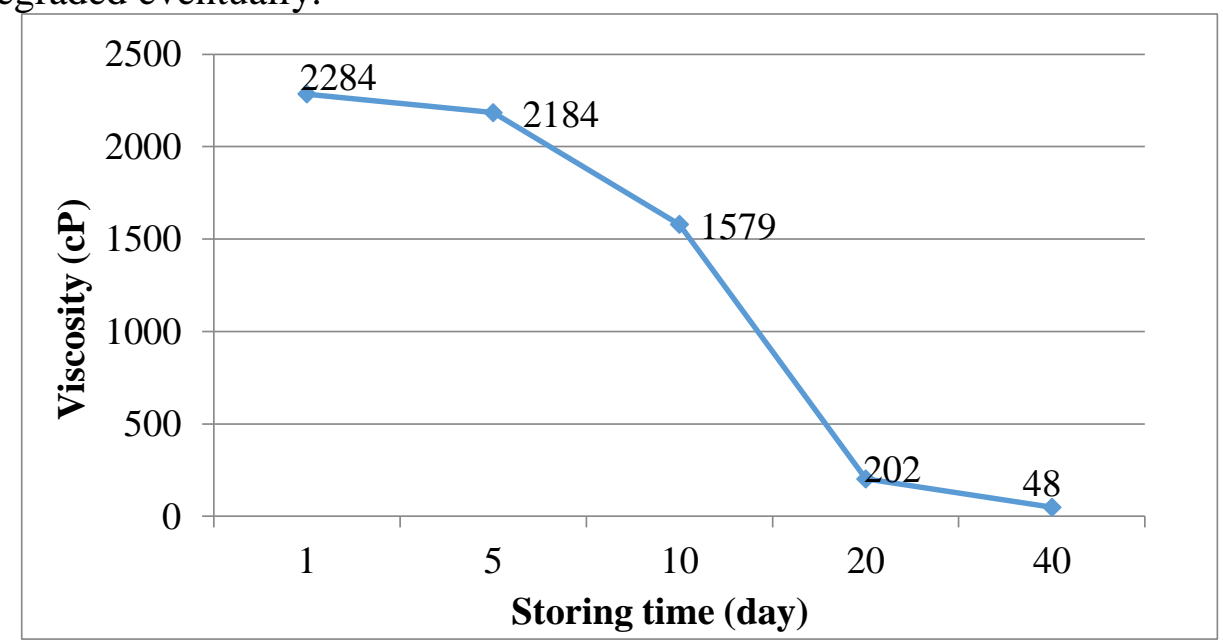

Figure 1: Storage time of spinning solution vs. viscosity

\section{Effect of age of solution on electrospinning}

The age of spinning solution significantly affects the electrospinning process. The freshly produced spinning solution creates frequent droplets about 5 minutes interval. Table 1 shows that up to a certain level the ageing of spinning solution positively affect electrospinning process - more the age of solution produces more stable fibre jet with less number of droplets. Previous study (Doshi and Reneker 1995) shows that the viscosity of PEO between 800 and $4000 \mathrm{cP}$ produce fibres, however, a viscosity lower than $800 \mathrm{cP}$ does not produce stable fibre jet. In this study, a stable fibre jet is obtained when solution viscosity is $2284 \mathrm{cP}$ at the age of day 1 but droplets were appeared on spinning solution. Hence, the solution of age of 1 day produces stable fibre jet with periodic droplets. However, more stable fibre jet without droplets were produced from the solution of day-5 and day-10 
whose viscosities were $2184 \mathrm{cP}$ and $1579 \mathrm{cP}$, respectively. Although the solution of age of 20 days whose viscosity was $202 \mathrm{cP}$ produced stable fibre jet but the solution of age of 40 days whose viscosity was only $48 \mathrm{cP}$ produced unstable and broken jet.

Table 1: Electrospinning process stability respect to age of spinning solution

\begin{tabular}{|c|c|c|c|c|}
\hline $\begin{array}{c}\text { Age of spinning } \\
\text { solution }\end{array}$ & $\begin{array}{c}\text { Working } \\
\text { distance }(\mathrm{cm})\end{array}$ & $\begin{array}{c}\text { Flow rate } \\
(\mathrm{ml} / \mathrm{h})\end{array}$ & $\begin{array}{c}\text { Applied } \\
\text { voltage }(\mathrm{kV})\end{array}$ & Observations \\
\hline 1-day & 16 & 0.3 & 12 & $\begin{array}{c}\text { Stable jet, droplet } \\
\text { formation }\end{array}$ \\
\hline 5-day & 16 & 0.3 & 12 & Stable jet \\
\hline 10-day & 16 & 0.3 & 12 & Stable jet \\
\hline 20-daye & 16 & 0.3 & 12 & Stable, very fine jet \\
\hline 40-day & 16 & 0.3 & 12 & Unstable very fine jet \\
\hline
\end{tabular}

\section{Effect of age of solution on morphological properties of fibres}

Solution viscosity has an immense effect on fibres morphology and diameter - lower viscosity is more likely to produce beaded fibres compare to higher viscosity solution. However, too much thick solution may retard the initiation of fibre jet. The 1 day old solution produced fibres with diameters from $98 \mathrm{~nm}$ to $256 \mathrm{~nm}$ where the average diameter of the fibres was $138 \mathrm{~nm}$ (Figure 2). Moreover, this solution produced some beads on the fibres. Hence, the average diameter and standard deviation (SD) of the fibres produced from 1-day solution is higher than the fibres produced from the 5day old solutions (Figure 2-3). The 5 days old solution produced comparatively lower fibres diameter along with lower standard deviation. This is because the viscosity of older solution is lower than new solution. Research also found that the higher viscosity produce coarser fibre diameter (Baumgarten 1971). However, the solution of day-20, and day-40 have been produced beaded fibres and the solutions of day- 5 and day-10 have been produced smoother fibres having least beads. The SEM images and fibre size distribution (Figure 2-6) show that the solutions of 5 and 10 days old produce fibres with best fibre properties.
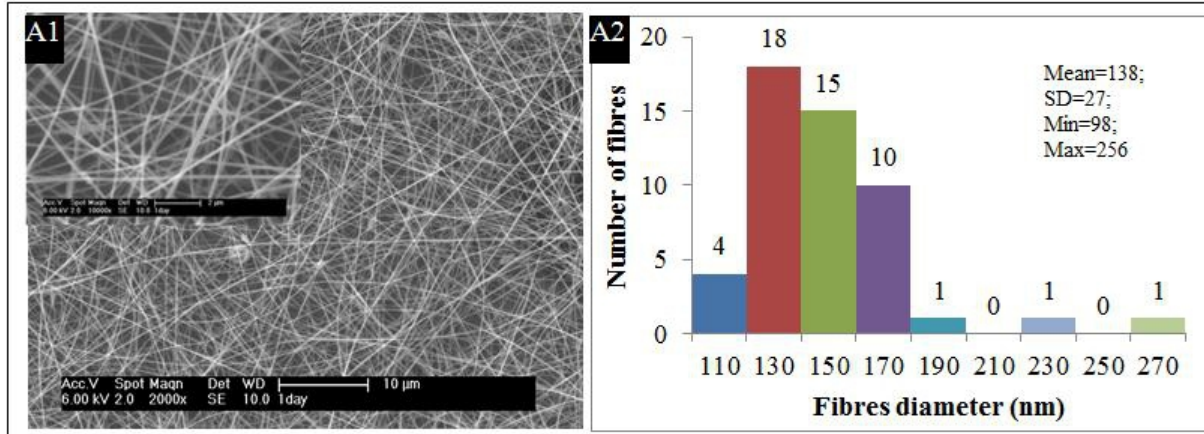

Figure 2: SEM image of 2000× (inset 10,000×) [A1] and fibre size distribution [A2] of Naalginate/PEO nanofibres obtained from 1-day spinning solution. 

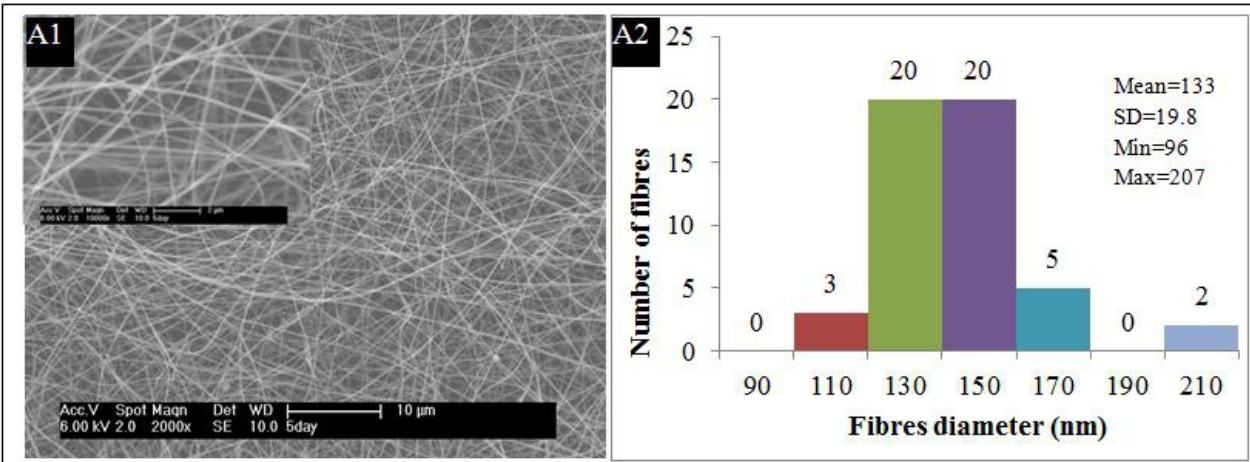

Figure 3: SEM image of $2000 \times($ inset $10,000 \times$ ) [A1] and fibre size distribution [A2] of Naalginate/PEO nanofibres obtained from 5-day spinning solution.
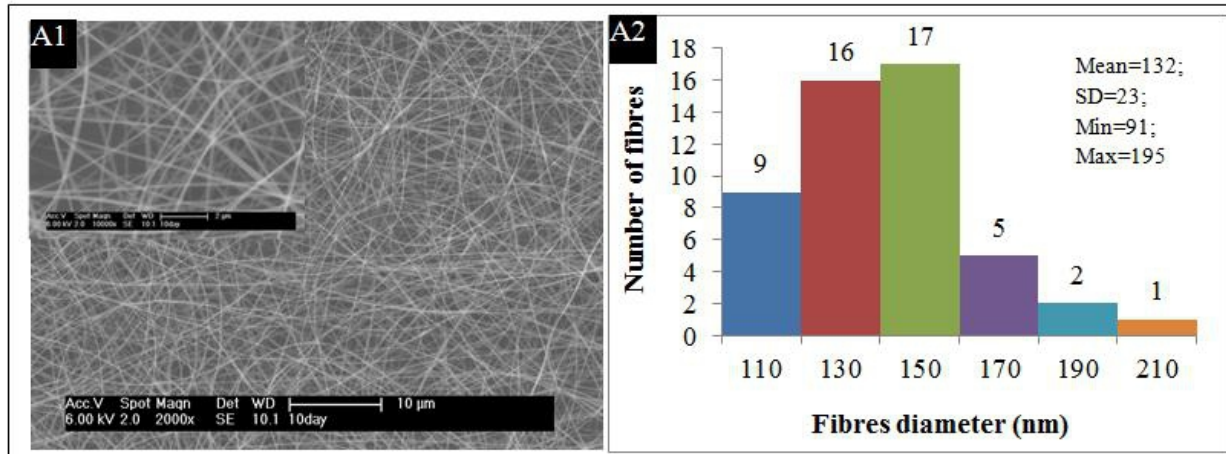

Figure 4: SEM image of $2000 \times($ inset $10,000 \times$ ) [A1] and fibre size distribution [A2] of Naalginate/PEO nanofibres obtained from 10-day spinning solution.

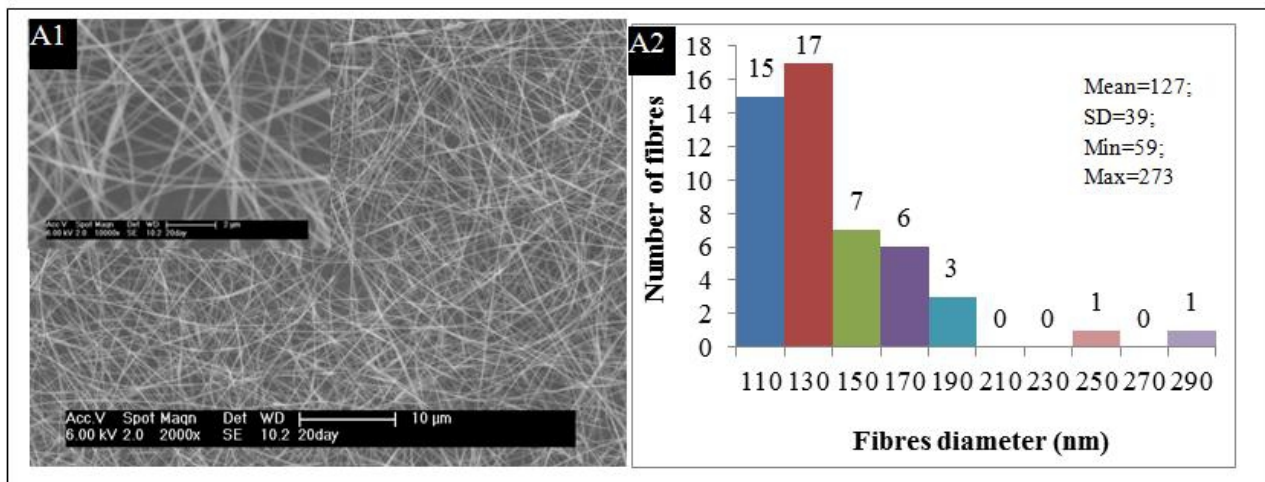

Figure 5: SEM image of 2000 $\times$ (inset 10,000 $\times$ ) [A1] and fibre size distribution [A2] of Naalginate/ PEO nanofibers obtained from 20-day spinning solution. 

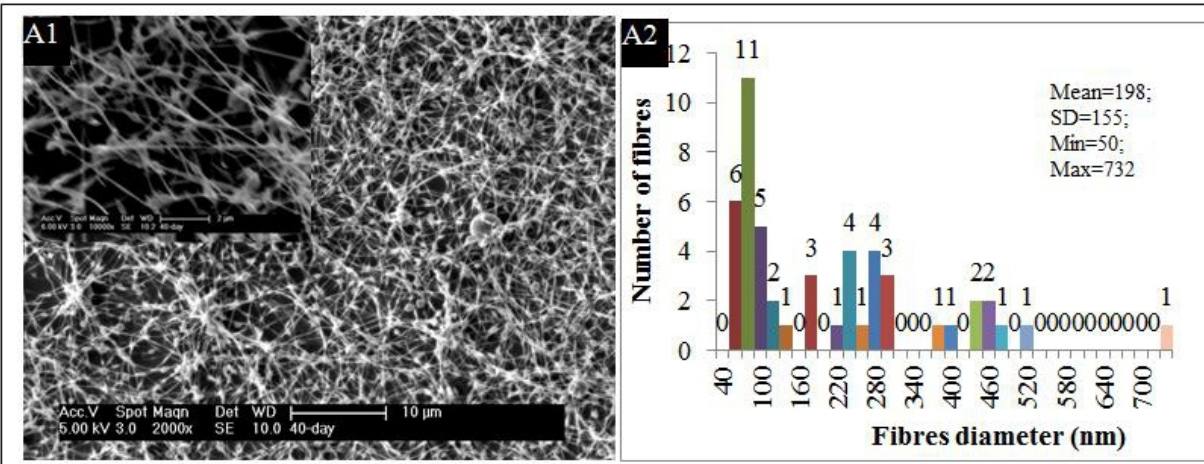

Figure 6: SEM image of $2000 \times$ (inset $10,000 \times$ ) [A1] and fibre size distribution [A2] of Naalginate/PEO nanofibres obtained from 40-day spinning solution.

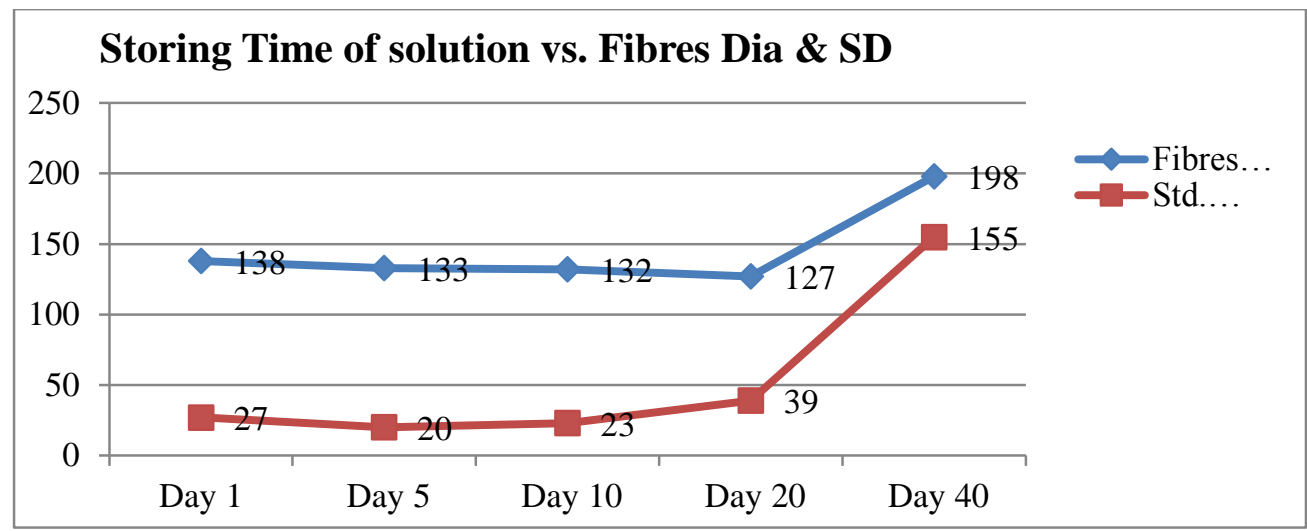

Figure 7: Age of solution vs. fibres diameter

\section{Effect of viscosity on morphological properties of fibres}

It has already been discussed that the viscosities of the Naalginate/PEO solution fall down when stored for prolonged period. This is obvious that the ageing of spinning solution shows an inverse relation with solution viscosity. It is the critical key in determining the fiber morphology. The storing time of polymer solution greatly affects the morphology and diameter of electrospun fibres, which has been discussed earlier. Hence, the fibres morphology and diameter are directly influenced by the storing time of the spinning solution. Figure 7 and 8 shows that the fibres mean diameters for the age of spinning solution of day-1, day-5, day-10 and day-20 are 138 $\mathrm{nm}, 133 \mathrm{~nm}, 132 \mathrm{~nm}, 127 \mathrm{~nm}$, respectively. Although the mean diameters of these fibres are close but the standard deviation varies considerably (Figure 7). The result shows that the diameters of the fibres yielded from the solution of day-5 and day-10 are more uniform. However, when spinning solution viscosity falls $48 \mathrm{cP}$ at day-40 a sudden augmentation of fibre average diameter occurs due to generation of beads on fibres. Fong et al. reported the 
consequence of bead formation in electrospun fibers occurs when the surface tension in the charged jet is sufficient to change the jet into droplets to reduce surface area (Fong et al. 1999). Therefore, it was hypothesized the lower viscosity of Na-alginate/PEO spinning solution was not able to fibre formation because of inadequate viscoelastic forces. So it is explicable that smooth and fine fibres with less number of beads yield from the $\mathrm{Na}$ alginate/PEO spinning solution of 5 to 10 days old.

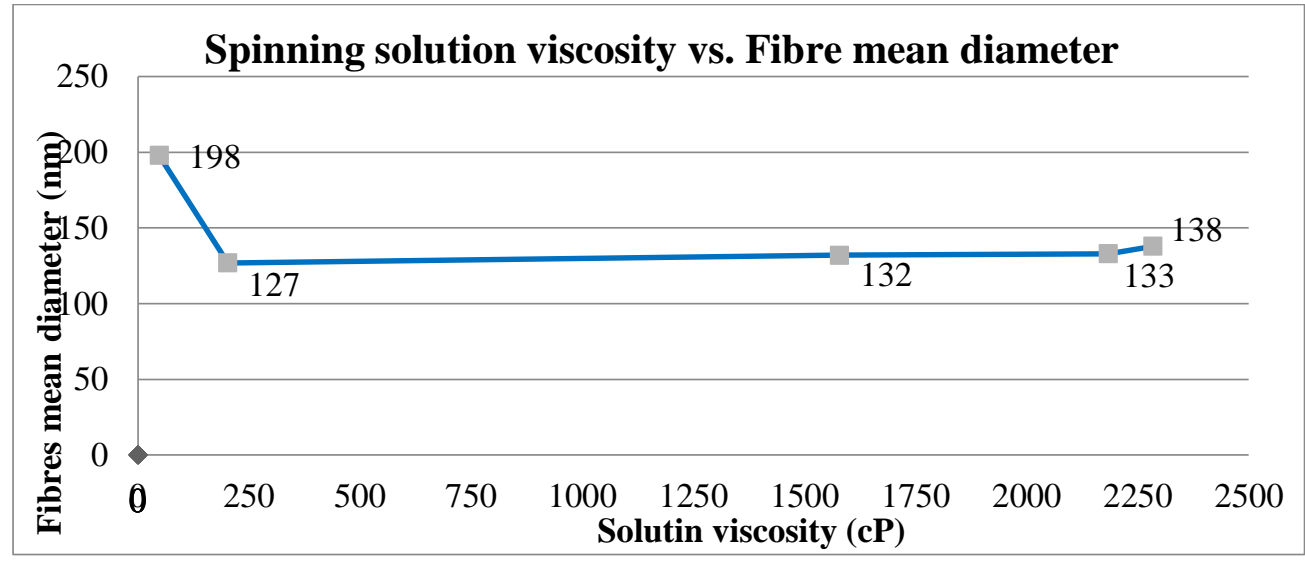

Figure 8: Viscosity of solution vs. fibres diameter

\section{FTIR analysis}

Fourier Transform Infrared Spectroscopy (FTIR) was used to characterize the Na-alginate/PEO nanofibes. Figure 9 shows the FTIR spectra of Na-alginate/PEO nanofibres within 4000-400 $\mathrm{cm}^{-1}$ (I) and 2000$1000 \mathrm{~cm}^{-1}$ (II) spectral range prepared using the solutions of different ages. The graph represents the spectral peak variations between the $\mathrm{Na}$ alginate/PEO nanofibres prepared using the solutions of the age of day-1, -5 , -10 , -20, and -40. An interaction is occurred between PEO and Na-alginate, which was confirmed by FTIR study. Usually, PEO shows some prominent spectrum at $1100 \mathrm{~cm}^{-1}$ and $843 \mathrm{~cm}^{-1}$ for $\mathrm{C}-\mathrm{O}-\mathrm{C}$ (ether group) asymmetric stretch and bending vibrations. On the other hand, Na-alginate provides some characteristic bands at $3336 \mathrm{~cm}^{-1}$ for $-\mathrm{OH}$ (hydroxyl group) stretching, 1593 $\mathrm{cm}^{-1}$ for COO- (carboxylate group) symmetrical stretching and $1410 \mathrm{~cm}^{-1}$ for COO- symmetrical stretching. Figure 9(i-B) shows that the asymmetrical absorption band for $\mathrm{C}-\mathrm{O}-\mathrm{C}$ of $\mathrm{PEO}$ was found at $1095 \mathrm{~cm}^{-1}$ in the $\mathrm{Na}$ alginate/PEO fibres due to the presence of $\mathrm{Na}$-alginate in the fibres. The asymmetrical band of $\mathrm{Na}$-alginate for $\mathrm{COO}^{-}$was shifted to $1612 \mathrm{~cm}^{-1}$ in Naalginate/PEO from $1593 \mathrm{~cm}^{-1}$. Moreover, the hydrogen bonds formed between ether oxygen of PEO and hydroxyl group of $\mathrm{Na}$-alginate in the $\mathrm{Na}$ alginate/PEO nanofibres, which is confirmed by the stretching band of $\mathrm{Na}$ alginate/PEO at $3336 \mathrm{~cm}^{-1}$. These interactions reduce the repulsive force 
between polyanionic sodium alginate molecules, which ultimately assists electrospinning. These results are consistent with previous studies (Nie et al. 2009; Lu et al. 2006; Jeong et al. 2010). Figure 1(II) shows a significant change in peaks of Na-alginate/PEO nanofibres prepared using the solutions of different ages at $2363 \mathrm{~cm}^{-1}$. According to the graph, this is clear that the change at $2363 \mathrm{~cm}^{-1}$ is occurred due to the degradation of Na-alginate over time. Na-alginate is significantly degraded after 5 days of storing time, and mostly degraded after 20 days.
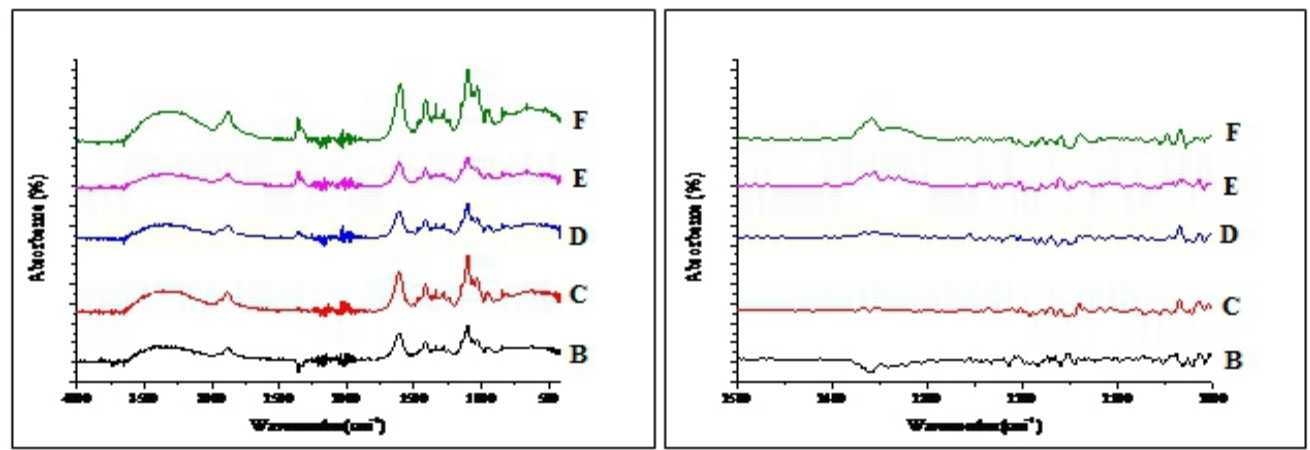

Figure 9: FTIR spectral curve for spinning solution of day-1 (B), day-5 (C), day-10 (D), day-20 (E) and day-40 (F).

\section{Conclusion}

Uniform nanofibres were obtained from the $4 \mathrm{wt} \%$ of $70: 30$ sodium alginate and poly(ethylene) oxide blend solution. The study investigated the effects of storing time of spinning solution on solution viscosity, fibre's diameters and morphology. The viscosity of the sodium alginate/PEO solution at the age of 1 day was found $2284 \mathrm{cP}$ which produced smooth and uniform nanofibres with an average diameter of $138 \mathrm{~nm}$. The viscosity of the spinning solution was reduced slowly over the time and found $2184 \mathrm{cP}$ at day 5 and $1579 \mathrm{cP}$ at day 10 . The corresponding solutions produced smooth and uniform nanofibres with average diameter of $133 \mathrm{~nm}$ and $132 \mathrm{~nm}$ as well as more stable fibre jets during electrospinning. However, the viscosity of the spinning solution was decreased significantly over the next days and found $202 \mathrm{cP}$ and $48 \mathrm{cP}$ at 20 days and 40 days, respectively. The solution of 40 days old yielded fibrous substance containing large beads, whereas the solution of 20 days old produced beaded fibres with high fluctuation of diameters.

\section{References:}

1. Baumgarten, P. K. (1971). Electrostatic spinning of acrylic microfibers. Journal of Colloid and Interface Science, 36(1), 71-79. 
2. Bhattarai, N., Li, Z., Edmondson, D. \& Zhang, M. (2006). AlginateBased Nanofibrous Scaffolds: Structural, Mechanical, and Biological Properties. Advanced Materials, 18(11), 1463-1467.

3. Cho, H. J., Yoo, Y. J., Kim, J. W., Park, Y. H., Bae, D. G. \& Um, I. C. (2012). Effect of molecular weight and storage time on the wetand electro-spinning of regenerated silk fibroin. Polymer Degradation and Stability, 97(6), 1060-1066.

4. Deitzel, J. M., Kleinmeyer, J., Harris, D. \& Beck Tan, N. C. (2001). The effect of processing variables on the morphology of electrospun nanofibers and textiles. Polymer, 42(1), 261-272.

5. Demir, M. M., Yilgor, I., Yilgor, E. \& Erman, B. (2002). Electrospinning of polyurethane fibers. Polymer, 43(11), 3303-3309.

6. Doshi, J. \& Reneker, D. H. (1995). Electrospinning Process and Applications of Electrospun Fibers. Journal of Electrostatics, 35(23), 151-160.

7. Fong, H., Chun, I. \& Reneker, D. H. (1999). Beaded nanofibers formed during electrospinning. Polymer, 40(16), 4585-4592.

8. ImageJ. (2004). ImageJ, National Institutes of Health, United States [Online]. Available: http://rsb.info.nih.gov/ij/index.html [Accessed 08/01/16].

9. Jarusuwannapoom, T., Hongrojjanawiwat, W., Jitjaicham, S., Wannatong, L., Nithitanakul, M., Pattamaprom, C., Koombhongse, P., Rangkupan, R. \& Supaphol, P. (2005). Effect of solvents on electro-spinnability of polystyrene solutions and morphological appearance of resulting electrospun polystyrene fibers. European Polymer Journal, 41(3), 409-421.

10. Kong, Q. S., Yu, Z. S., Ji, Q. \& Xia, Y. Z. (2009). Electrospinning of Sodium Alginate with Poly(ethylene oxide), Gelatin and Nanometer Silver Colloid. Materials Research, Pts 1 and 2, 610-613, 1188-1191.

11. Li, L. \& Hsieh, Y. L. (2006). Chitosan bicomponent nanofibers and nanoporous fibers. Carbohydrate Research, 341(3), 374-381.

12. Mincheva, R., Manolova, N., Paneva, D. \& Rashkov, I. (2005). Preparation of polyelectrolyte-containing nanofibers by electrospinning in the presence of a non-ionogenic water-soluble polymer. Journal of Bioactive and Compatible Polymers, 20(5), 419435.

13. Poursorkhabi, V., Mohanty, A. K. \& Misra, M. (2015). Electrospinning of Aqueous Lignin/Poly(ethylene oxide) Complexes. Journal of Applied Polymer Science, 132(2).

14. Reneker, D. H. \& Chun, I. (1996). Nanometre diameter fibres of polymer, produced by electrospinning. Nanotechnology, 7(3), 216223. 
15. Saquing, C. D., Tang, C., Monian, B., Bonino, C. A., Manasco, J. L., Alsberg, E. \& Khan, S. A. (2013). Alginate-Polyethylene Oxide Blend Nanofibers and the Role of the Carrier Polymer in Electrospinning. Industrial \& Engineering Chemistry Research, 52(26), 8692-8704.

16. Son, W. K., Youk, J. H., Lee, T. S. \& Park, W. H. (2004). The effects of solution properties and polyelectrolyte on electrospinning of ultrafine poly(ethylene oxide) fibers. Polymer, 45(9), 2959-2966. 